\title{
The simple method of dynamic visco-elastic analysis of road structure on rheological foundation
}

\author{
Sławomir Karaś, Magdalena Sawecka \\ Road and Bridge Department, Lublin University of Technology
}

\begin{abstract}
In contrast to computationally advanced methods of road pavement dynamic analysis, the one-dimensional, simple method is derived on the basis of visco-elastic simple beam lying on generalized Winkler visco-elastic foundation. By virtue of least square method the visco-elastic constants could be estimated with technically admissible accuracy. The introduced method is useful enough to predict any pavement deformation process in the range of linear visco-elasticity.
\end{abstract}

Key words: road pavement, visco-elasticity, rheology.

\section{Introduction}

The presented here range of investigation is limited to the theoretical part of the task, i.e. the calibration to real load and structures is not yet covered.

The aim of the paper is a proposal of a simple procedure for estimating the visco-elastic characteristics by means of initial-boundary problem of a simple beam laid on flexible foundation, where both beam and foundation, have visco-elastic properties. The simplicity of the approach is crucial, which is in contrast to the computationally intensive method, i.e. for example Veroad [1, 2].

The research of the material constants in road engineering has an extended bibliographical background, including hundreds of papers in which both theoretical and experimental studies were undertaken. Historically, the first step could be given for those who applied the Burmister method [2, 3], which obeys the multilayered elastic semi-space subjected to concentrated load. This involves the axially symmetric problem in cylindrical coordinates. On this basis the so called 'backcalculating methods' of pavement modulus are still in use [4-14]. As an example of such work, and as an example of almost exact accuracy, the paper of S. Firlej should be recalled [15].

The effective visco-elastic approaches to the pavement mechanics are not scarce [16-17] but, on the other hand, not intensive enough. An extension to visco-elastic Boussinesq problem could be found in [8]. The survey of visco-elastic foundation types and an original concept of elastic beam on Maxwell foundation is analyzed in Szcześniak's work [19], where the concentrated force moves with the constant velocity along the beam.

\section{The formulation of the problem}

The mineral-asphalt materials of road pavements have elastic, viscous and plastic properties, but due to the serviceability of the road structures the first two of 
them are of great importance. Although, the plastic behavior is taken into consideration, its effect generates the development of disadvantageous deflections and wheel tracks. The value of complex modulus is the basic criterion to classify the pavement material in the range of its usability for the road structure. Optionally, one can assume that the live service of the pavement is connected with its elastic and viscous properties. The analyzed here pavement model obeys the generalized Kelvin-Voigt relation:

$$
\sigma=E \varepsilon+\hat{E} \dot{\varepsilon}+\bar{E} \ddot{\varepsilon},
$$

where $E-$ is an elastic modulus and $\hat{E}, \bar{E}$ are the viscous moduli, $\sigma-$ stress, $\varepsilon-$ strain and $\dot{\varepsilon}, \ddot{\varepsilon}$ are rates of strain and rates of strain rate adequately.

The road embankment or, in detail the road subgrade, could be treated as a generalization of Winkler foundation extended for viscous properties, for which the no-unilateral i.e. negative as well as positive constrains, are admissible. The parameters of generalized Winkler model are: $\left.k \mathrm{kN} / \mathrm{m}^{2}\right]$ - elastic settlement coefficient and $\eta\left[\mathrm{Ns} / \mathrm{m}^{3}\right]$ - the coefficient of viscosity.

The differential equation of the problem has the following form:

$$
E J\left(\frac{\partial^{4} u}{\partial x^{4}}+\hat{\psi} \frac{\partial^{4} \dot{u}}{\partial x^{4}}+\bar{\psi} \frac{\partial^{4} \ddot{u}}{\partial x^{4}}\right)+\rho \ddot{u}+k u+\eta \dot{u}=q,
$$

where: $u=u(x, t)$ - is the beam deflection, $0 \leq x \leq L-$ abscissa domain, $t$ - time parameter, $E J$ - bending stiffness of the beam, $\rho$ - beam mass per unit length distribution, $q=q(x, t)$ - is linear density of loads, constants:

$$
\hat{\psi}=\hat{E} / E, \bar{\psi}=\bar{E} / E .
$$

Introducing the dimensionless description by means of

$$
\begin{aligned}
& \xi=x / L, \quad 0 \leq \xi \leq 1, \omega=u / L, \tau=t / t_{0}, \quad 0 \leq \tau \leq \infty, \\
& t_{0}{ }^{2}=\frac{\rho L^{4}}{E J}, \quad c=\frac{k L^{4}}{E J}, \hat{c}=\frac{\eta L^{4}}{t_{0} E J}, q_{1}=\frac{q L^{3}}{E J}, \hat{\chi}=\frac{\hat{\psi}}{t_{0}}, \bar{\chi}=\frac{\bar{\psi}}{t_{0}{ }^{2}},
\end{aligned}
$$

we arrive at:

$$
\frac{\partial^{4}}{\partial \xi^{4}}(\omega+\hat{\chi} \dot{\omega}+\bar{\chi} \ddot{\omega})+\ddot{\omega}+\hat{c} \cdot \dot{\omega}+c \cdot \omega=q_{1} .
$$

To simplify the task, the initial conditions are uniform and for each function at a moment $\tau=0$ are zeros.

In technical sense the influence of beam boundary conditions, when the beam laid on flexible foundation, could be neglected when its supports are localized far from the analyzed cross-section. Due to this the length of a beam is considerably greater than the vehicle length. According to this the simple beam is used for problem modeling. 


\section{Green function}

Assuming now that we are looking for Green function of the problem the following form of the load is analyzed:

$$
q_{1}:=Q=\delta\left(\xi-\xi_{0}\right) \delta\left(\tau-\tau_{0}\right) \rightarrow \omega(Q):=G_{\omega}\left(\xi, \xi_{0}, \tau, \tau_{0}\right),
$$

hence:

$$
\omega(\xi, \tau)=\int_{0}^{\tau} \int_{0}^{1} G_{\omega}\left(\xi, \xi_{0}, \tau, \tau_{0}\right) \cdot Q\left(\xi_{0}, \tau_{0}\right) d \xi_{0} d \tau_{0},
$$

where: $\delta-$ is the symbol of Dirac impulse function.

Using operational calculus, i.e. finite Fourier transformation according to geometrical parameter $\xi$ and Laplace transformation onto time parameter $\tau$, the solution is obtained in the form of the following sum

$$
G_{\omega}\left(\xi, \xi_{o}, \tau, \tau_{o}\right)=\sum_{m=1}^{3} G_{\omega_{m}},
$$

where $G_{\omega_{1}}, G_{\omega_{2}}, G_{\omega_{3}}$ have the appropriate forms depending on the value of $\Delta=\Delta(n)$ indicator as follows:

$$
\begin{aligned}
& A=\bar{\chi}(n \pi)^{4}+1 \\
& \Delta=B^{2}-4 A C, \quad B=\hat{\chi}(n \pi)^{4}+\hat{c}, \quad a=\frac{-B}{2 A}, \\
& C=(n \pi)^{4}+c \\
& b=\frac{\sqrt{4 A C-B^{2}}}{2 A}, \quad d=\frac{\sqrt{B^{2}-4 A C}}{2 A}, \\
& \Delta(n, \hat{\psi}, \bar{\psi}, \hat{c})=\left(\frac{\hat{\psi}}{t_{o}}(n \pi)^{4}+\hat{c}\right)^{2}-4\left(\frac{\bar{\psi}}{t_{o}^{2}}(n \pi)^{4}+1\right)\left((n \pi)^{4}+c\right),
\end{aligned}
$$

and for:

1. $\Delta>0 \rightarrow n_{1}$ :

$$
G_{\omega 1}=2 \cdot \sum_{n=n_{1}} \frac{e^{a\left(\tau-\tau_{o}\right)}}{A d} \sinh \left(d\left(\tau-\tau_{o}\right)\right) \sin \left(n \pi \xi_{o}\right) \cdot \sin (n \pi \xi) ;
$$

2. $\Delta=0 \rightarrow n_{2}$ :

$$
G_{\omega 2}=2 \cdot \sum_{n=n_{2}} \frac{a e^{a\left(\tau-\tau_{o}\right)}}{A} \sin \left(n \pi \xi_{o}\right) \cdot \sin (n \pi \xi) ;
$$

3. $\Delta<0 \rightarrow n_{3}$ :

$$
G_{\omega 3}=2 \cdot \sum_{n=n_{3}} \frac{e^{a\left(\tau-\tau_{o}\right)}}{A b} \sin \left(b\left(\tau-\tau_{o}\right)\right) \sin \left(n \pi \xi_{o}\right) \cdot \sin (n \pi \xi) .
$$




\section{The concentrated load}

The Green function directly corresponds to the case of concentrated load, as it is shown in Fig. 1.

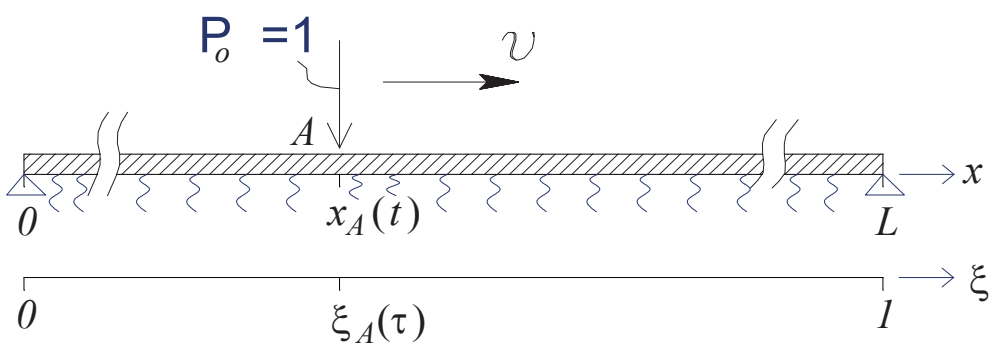

Fig. 1. The load in the form of concentrated force and its localization.

The load satisfies the following definition:

$$
q_{1}(\xi, \tau)=\left\{\begin{array}{ccc}
P_{1} \cdot \delta\left(\xi-\mathbf{v}_{1} \cdot \tau\right) & \text { for } & \tau \in\left\langle 0 ; \tau_{k}\right\rangle \\
0 & \text { if } & \tau>\tau_{k}
\end{array},\right.
$$

where: $\tau_{k}=1 / \mathbf{v}_{1}, \quad \mathbf{v}_{1}=\mathbf{v} t_{o} / L$.

Hence, the beam displacement process is described in form (8) by means of sum elements:

1. $\Delta>0$ :

$\omega_{1}(\xi, \tau)= \begin{cases}\sum_{n_{1}} C_{1} \int_{0}^{\tau} e^{a\left(\tau-\tau_{o}\right)} \sinh \left(d\left(\tau-\tau_{o}\right)\right) \sin \left(n \pi \mathbf{v}_{1} \cdot \tau_{o}\right) d \tau_{o} & \text { for } \quad \tau \in\left\langle 0 ; \tau_{k}\right\rangle \\ \sum_{n_{1}} C_{1} \int_{0}^{\tau_{k}=\frac{1}{\mathbf{v}_{1}}} e^{a\left(\tau-\tau_{o}\right)} \sinh \left(d\left(\tau-\tau_{o}\right)\right) \sin \left(n \pi \mathbf{v}_{1} \cdot \tau_{o}\right) d \tau_{o} & \text { if } \quad \tau>\tau_{k}\end{cases}$

where: $C_{1}=\frac{2 P_{1} \sin (n \pi \xi)}{A d}$;

2. $\Delta=0$ :

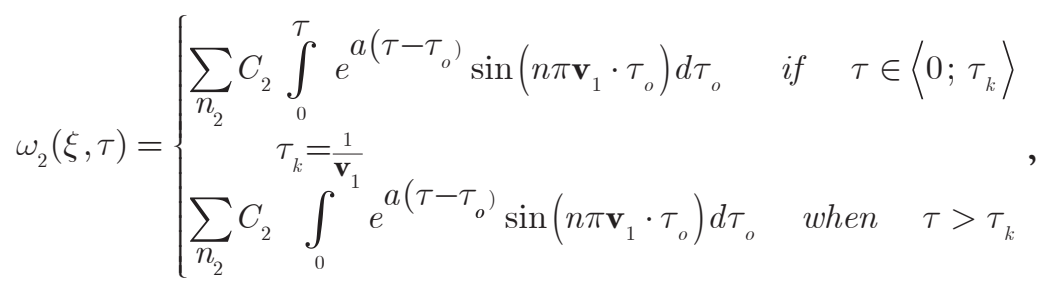

where: $C_{2}=\frac{2 P_{1} a \sin (n \pi \xi)}{A}$;

3. $\Delta<0$ : 


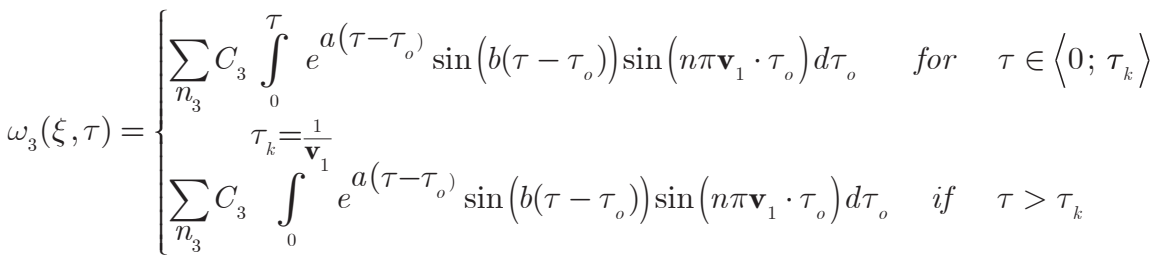

where: $C_{3}=\frac{2 P_{1} \sin (n \pi \xi)}{A b}$.

The constants $a, b, d, A, B, C$ - are given by (9) formulae.

\section{Axial load}

In computational practice the action of a single wheel is basic, Fig. 2. For this variant the load contact area has the length of $30 \mathrm{~cm}$, and is relatively small comparing to the beam span.

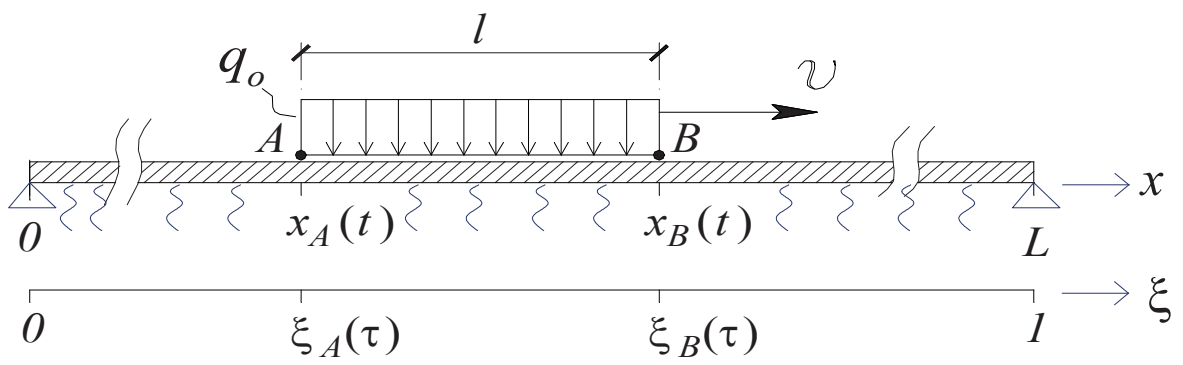

Fig. 2. Sectorially uniformly distributed movable load.

The terminal abscissas have the following form:

$$
x_{A}(t)=\mathbf{v} \cdot t, \quad x_{B}(t)=\mathbf{v} \cdot t+l,
$$

hence we have:

$$
\xi_{A}(\tau)=\mathbf{v}_{1} \cdot \tau, \quad \xi_{B}(\tau)=\mathbf{v}_{1} \cdot \tau+l / L,
$$

where: $\mathbf{v}_{1}=\left(\mathbf{v} t_{o}\right) / L$.

The load function fulfills the expression:

$$
q(\xi, \tau)=q_{o} \cdot\left[\eta\left(\xi-\xi_{A}(\tau)\right)-\eta\left(\xi-\xi_{B}(\tau)\right)\right]
$$

where: $\tau \in\left(0, \tau_{k}=1 / \mathbf{v}_{1}\right)$ and $q_{o 1}=\frac{q_{o} L^{3}}{E J}$.

The solution has the form (8), while sum elements are listed below:

1. $\Delta>0$ : 


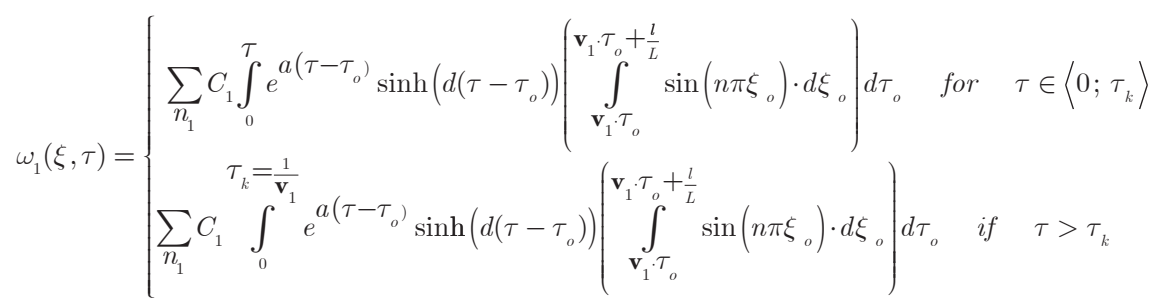

where: $C_{1}=\frac{2 q_{o 1} \sin (n \pi \xi)}{A d}$;

2. $\Delta=0$ :

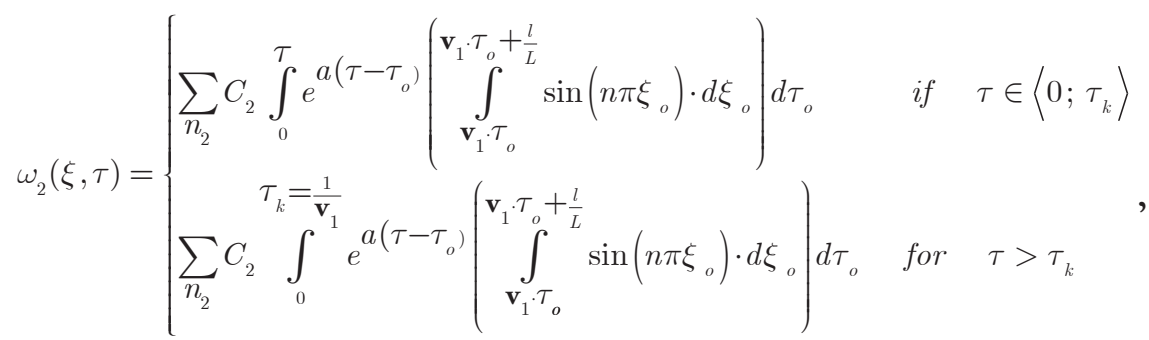

where: $C_{2}=\frac{2 q_{01} a \sin (n \pi \xi)}{A}$;

3. $\Delta<0$ :

$\omega_{3}(\xi, \tau)=\left\{\begin{array}{l}\sum_{n_{3}} C_{3} \int_{0}^{\tau} e^{a\left(\tau-\tau_{o}\right)} \sin \left(b\left(\tau-\tau_{o}\right)\right)\left(\int_{\mathbf{v}_{1} \cdot \tau_{o}}^{\mathbf{v}_{1} \cdot \tau_{o}+\frac{l}{L}} \sin \left(n \pi \xi_{o}\right) \cdot d \xi_{o}\right) d \tau_{o} \quad \text { for } \quad \tau \in\left\langle 0 ; \tau_{k}\right\rangle \\ \sum_{n_{3}} C_{3} \int_{0}^{\tau_{k}=\frac{1}{\mathbf{v}_{1}}} e^{a\left(\tau-\tau_{o}\right)} \sin \left(b\left(\tau-\tau_{o}\right)\right)\left(\int_{\mathbf{v}_{1} \cdot \tau_{o}}^{\mathbf{v}_{1} \cdot \tau_{o}+\frac{l}{L}} \sin \left(n \pi \xi_{o}\right) \cdot d \xi_{o}\right) d \tau_{o} \quad \text { if } \quad \tau>\tau_{k}\end{array}\right.$

where: $C_{3}=\frac{2 q_{o 1} \sin (n \pi \xi)}{A b}$.

Supplementing the above visco-elastic solution, an elastic variant obeying the wheel setting in the middle of the span at a time moment $t=0$ is derived, hence we arrive at:

$$
q_{1}(\xi, \tau)=q_{o 1} \cdot\left[\eta\left(\xi-\xi_{A}\right)-\eta\left(\xi-\xi_{B}\right)\right] \eta(\tau),
$$

where: $\xi_{A}=0.5\left(1-\frac{l}{L}\right), \xi_{B}=0.5\left(1+\frac{l}{L}\right)$,

which implies:

$$
\omega(\xi, \tau)=\sum_{n=1}^{\infty} \frac{2 q_{o 1} \sin (n \pi \xi)}{\beta_{n}{ }^{2} \cdot n \pi}\left(\cos \left(n \pi \xi_{A}\right)-\cos \left(n \pi \xi_{B}\right)\right)\left(1-\cos \left(\beta_{n} \tau\right)\right) ;
$$


if: $\quad \beta_{n}=\sqrt{n^{4} \pi^{4}+c}, \quad c=\frac{k L^{4}}{E J}$.

\section{Numerical examples}

The dynamical and visco-elastic characteristics of the road structure are unknown in general. However, on the basis of different bibliographical sources or carried out tests, it is possible to estimate those values as good enough approximations. We can also search them in an exact procedure applying least square methods additionally aided with robust procedures, or get them treating elastic constants as an initial approximation in iteratively refining loops.

In this paper such approximating values are assumed to demonstrate the efficiency of the introduced theory only.

The input values (for two asphalt-concrete pavement layers we have):

- upper layer of a thickness $5 \mathrm{~cm}, \mathrm{E}=18500 \mathrm{MPA}$,

- bottom layer of the thickness $7 \mathrm{~cm}, \mathrm{E}=18000 \mathrm{MPA}$,

- base of $20 \mathrm{~cm}$ crushed stone $\mathrm{E}=400 \mathrm{MPa}$;

- those together give $\Sigma 32 \mathrm{~cm}$, and weighted average modulus $E_{a v .} \approx 7080$ $\mathrm{MPa}$.

The elastic modulus value obtained by means of Burmister method [4], when the subgrade modulus is $E_{0}=100 \mathrm{MPa}$, for such layers set has the magnitude of $1700 \mathrm{MPa}$. The equivalent modulus value is equal to $E_{\text {eq. }} \cong 450 \mathrm{MPa}$, and then the elastic maximum flexure attain $u_{B-e} \cong 0,41 \mathrm{~mm}$.

For further computation, for the beam the value $\mathrm{E}=1700 \mathrm{MPa}$ is assumed.

Geometrical characteristics of a beam are as follows:

- $\operatorname{span}-L=30 \mathrm{~m}$,

- height $-h=0,32 \mathrm{~m}$,

- breath $-b=1 \mathrm{~m}$,

- principal second moment of area $J=b h^{3} / 12 \mathrm{~m}^{4}$.

Material visco-elastic characteristics:

- $\hat{\psi}=0,01, \bar{\psi}=0,001$,

- beam material weighted average density $\rho_{0}=2200 \mathrm{~kg} / \mathrm{m}^{3}$,

- and its linear density $\rho=\rho_{0} b h=6875 \mathrm{~kg} / \mathrm{m}$.

Load characteristics:

- velocity: $V=50 \mathrm{~km} / \mathrm{h}=13,89 \mathrm{~m} / \mathrm{s}$,

- load magnitude $q_{0}=\frac{P_{0}}{0,3}=\frac{0,65 \pi(0,3)^{2}}{4 \cdot 0,3} \mathrm{MN} / \mathrm{m}$,

- Winkler elastic ground parameter $k=150 \mathrm{MN} / \mathrm{m}^{3}$,

- ground viscous coefficient $\eta=0$.

Let us start with the comparison of Burmister method to the one introduced here. In conformity to elasticity, the zero values of $\hat{\psi}=\bar{\psi}=k=0$ are necessary. 
Using (17) we can obtain the elastic deformation of beam midspan cross-section for any time moment, starting from the beginning of the process, i.e. from the moment when the wheel is put down in the midspan. The result for $\tau=0.0005$ is shown below in Fig. 3.

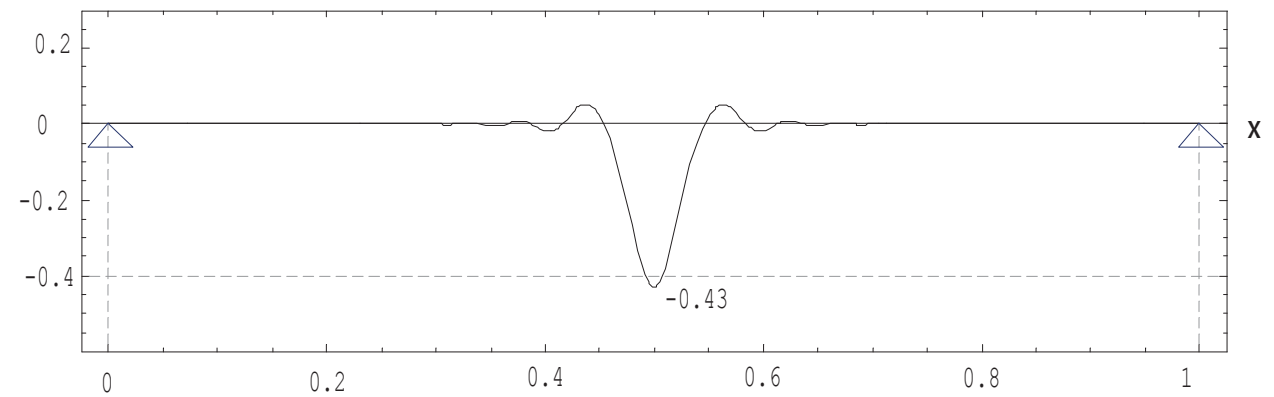

Fig. 3. The elastic displacement value $u=0,43 \approx u_{B-e} \mathrm{~mm}$.

From now on the results will concern the visco-elastic properties of beam material i.e. $\hat{\psi}=0,01, \bar{\psi}=0,001$, for $q_{0} l$ uniform load distribution. The history of midspan cross-section vertical displacement is shown in Fig. 4.

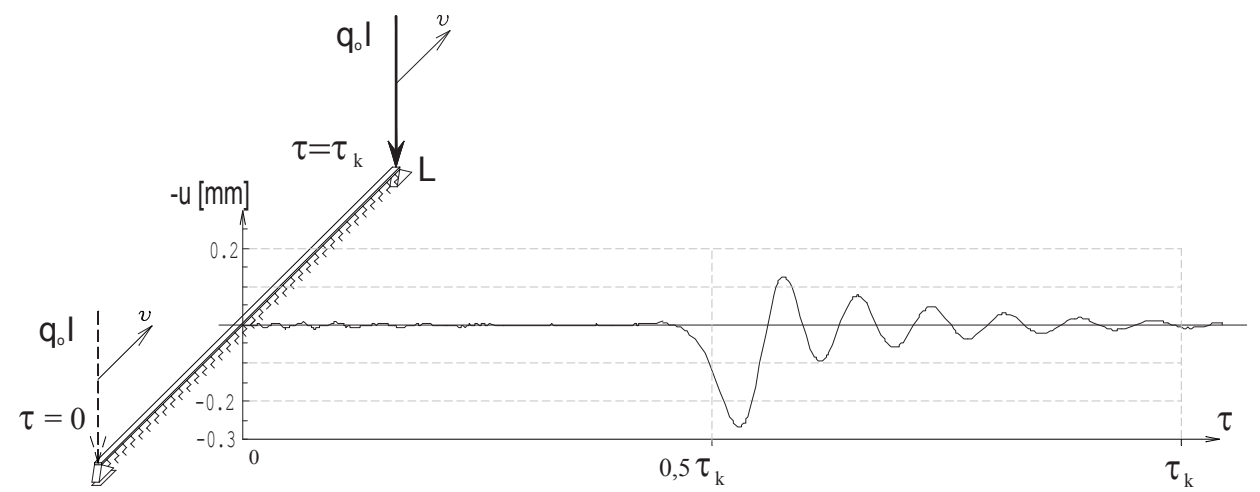

Fig. 4. The graph of the beam midspan point displacement from 0 to $\tau_{k}$ of $q_{0} l$ movement.

The graphs of beam deformation states are shown in Fig. 5-7 for $\tau=0,25 \tau_{k}$, $\tau=0,75 \tau_{k}, \tau=1,1 \tau_{k}$.

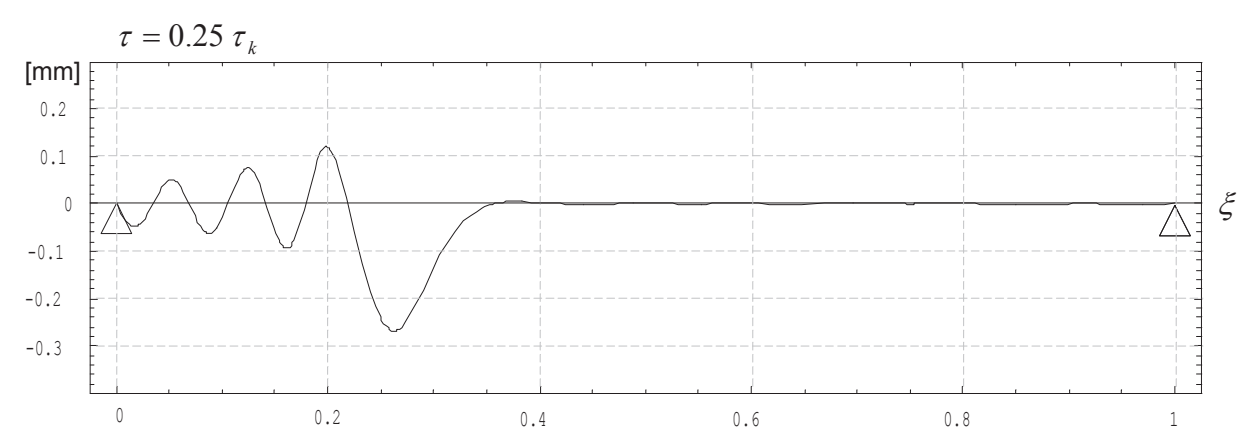

Fig. 5. Deflection at $\tau=0,25 \tau_{k}$. 


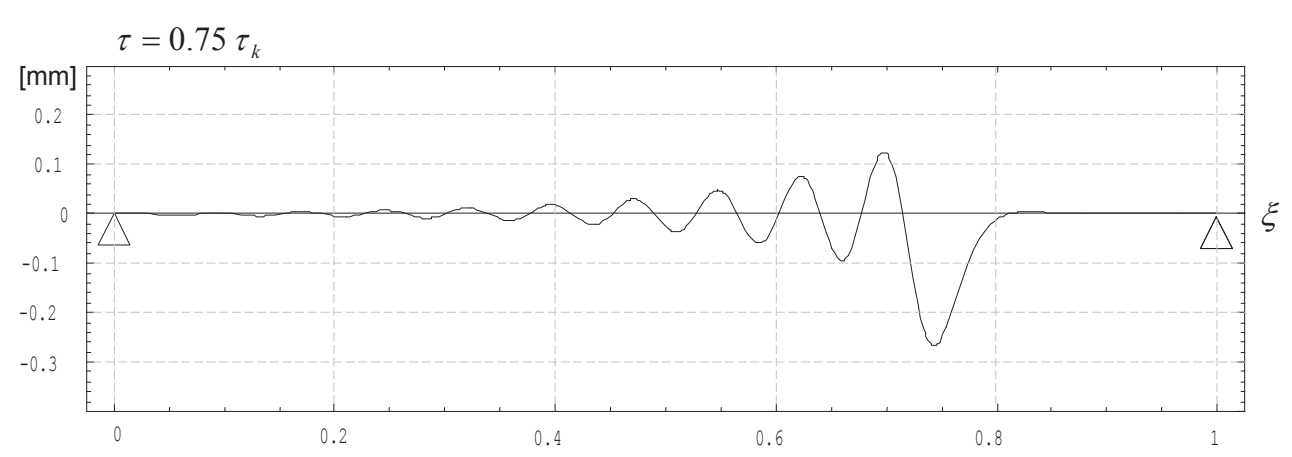

Fig. 6. Deflection at $\tau=0,75 \tau_{k}$.

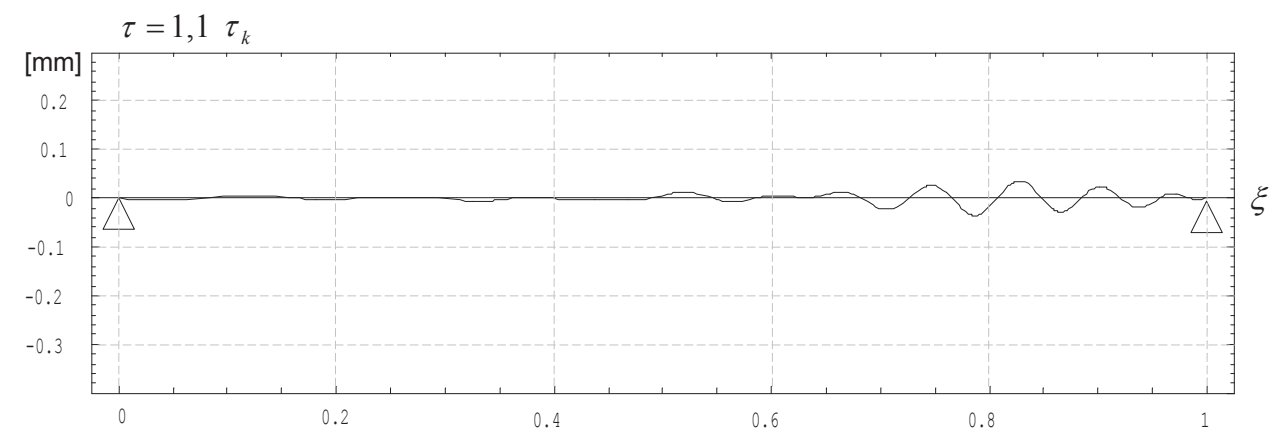

Fig. 7. Deflection at $\tau=1,1 \tau_{k}$.

As a result of carried out comparative calculations it is possible to notice that the difference between $q_{0} l$ and $P_{0}$ actions is negligible and as a consequence instead of (16) the (13) formulae could be used as a model of vehicle wheel.

\section{Conclusions}

Comparatively simple one-dimensional model of road pavement visco-elastic flexion process has been derived, and its usefulness is shown. The numerical examples were executed by Mathematica.

The linear visco-elastic theory is assumed by virtue of statement that the plastic behavior, and as an effect the disqualifying deformations are caused due to inadmissible overloading of road structure. Mathematical linearity converges with superposition in mechanics. This allows summing on both actions and effects sides. Further this implies that an arbitrary type of vehicle could be chosen to the experimental test.

On the basis of existing bibliography or the results of elastic approaches [20], the elastic constants could be taken as known, the other viscous material constants i.e. $\eta, \hat{\psi}, \bar{\psi}$ could be found by means of least square method or its variants. Disposing of a set of experimental data:

$$
u_{(m)}=u_{(m)}\left(x_{(m)} t_{(m)}\right) \rightarrow \omega_{(m)}=\omega_{(m)}\left(\xi_{(m)} \tau_{(m)}\right)
$$

we can write down: 


$$
\Sigma=\sum_{m=1}^{\bar{m}}\left[\omega\left(\xi_{(m)} \tau_{(m)}\right)-\omega_{(m)}\left(\xi_{(m)} \tau_{(m)}\right)\right]^{2}=\min .
$$

where: $\omega\left(\xi_{(m)} \tau_{(m)}\right)$ is taken in (13) or (16) form. The necessary conditions of $\Sigma$ extremum are as follows:

$$
\frac{\partial \Sigma}{\partial \eta}=0, \frac{\partial \Sigma}{\partial \hat{\psi}}=0, \frac{\partial \Sigma}{\partial \bar{\psi}}=0,
$$

which form the three non-linear equation system for $\eta, \hat{\psi}, \bar{\psi}$ determination.

\section{References}

[1] Nilsson RN, Oost I, Hopman PC, Visco-elastic analysis of full scale pavements: validation of VEROAD, Transportation research record, 1539, TRB. Washington, DC: National Research Council; 1996 p. 81-87.

[2] Hopman P., Nilsson R., Pronk A., Theory, validation and application of the visco-elastic multilayer program VEROAD, ICAP, Seattle, USA 1997.

[3] Burmister DM., The theory of stresses and displacements in layered systems and applications to the design of airport runways, Proceedings of Highways Research Board; 23: 127-48, 1943.

[4] Burmister DM., The general theory of stresses and displacements in layered soil systems, Journal of Applied Physics; 16: 89-94, 126-127; 296-302, 1945.

[5] Sivaneswaran N, Kramer SL, Mahoney JP., Advanced backcalculation using a nonlinear least squares optimization technique, Transportation research record, 1293, TRB. Washington, DC: National Research, Council; 1991 p. 93-102.

[6] Chatti K, Kim T., Effect of frequency-dependent asphalt concrete Layer moduli on pavement response, In: Tayabji SD, Lukanen EO, editors, NDT of pavements and backcalculation of moduli, 3. Special technical publication, STP 1375. Pennsylvania: ASTM Publication; 2000. p. 365-82.

[7] Briggs RC, Lukanen EO., Variations in backcalculated pavement layer moduli in LTTP seasonal monitoring sites. In: Tayabji SD, Lukanen EO, editors, NDT of pavements and backcalculation of moduli, 3. Special technical publication, STP 1375. Pennsylvania: ASTM Publication; 2000. p. 113-28.

[8] Zhou H., Comparison of backcalculated and laboratory measured moduli on AC and granular base layer materials, In, Tayabji SD, Lukanen EO, editors, NDT of pavements and backcalculation of moduli, 3. Special technical publication, STP 1375, vol. 3-37. Pennsylvania: ASTM Publication; 2000. p. 161-72.

[9] Fleming PR., Small-scale dynamic devices for the measurement of elastic stiffness modulus on pavement foundations, In: Tayabji SD, Lukanen EO, editors, Nondestructive testing of pavements and backcalculation of moduli, 3. Special technical publication, STP 1375. Pennsylvania: ASTM Publication; 2000. p. 41-58.

[10] Reddy MA, Reddy KS, Pandey BB., Backcalculation of pavement moduli using genetic algorithms, J Highw Res Board 2002; 66:1-10.

[11] Liang R, Zeng S., Efficient dynamic analysis of multilayered system during falling weight deflectometer experiments, J Transp Eng ASCE 2002; 128(4): 366-74.

[12] Dong QX, Hachiya Y, Takahashi O, Tsubokawa Y, Matsui K., An efficient backcalculation algorithm of time domain for large-scale pavement structures using Ritz vectors, Finite Elem Anal Des 2002; 38: 1131-50. 
[13] Terzi S, Saltan M, Yildirim T., Optimization of deflection basin by genetic algorithm and neural network approach, International Conference on Neural Networks, ICANN/ ICONIP 2003, LNCS 2714, 2003: 662-669.

[14] Reddy MA, Reddy KS, Pandey BB., Selection of genetic algorithm parameters for backcalculation of pavement moduli, Int J Pavement Eng 2004; 5(2): 81-90.

[1]5 Firlej S., Inaccuracy in back-calculating the E-moduli of road pavements, in edited by J.J. Luchko Fracture Mechanics and Physics of Construction Materials and Structures, 575-584, Collection of Scientific Works, 6, Lviv, 2005.

[16] Sousa JB, Weissman SL, Sackman JL, Monismith CL., Nonlinear elastic viscous with damage model to predict permanent deformation of asphalt concrete mixes, Transportation research record, 1136, TRB. Washington, DC: National Research Council; 1993 p. 57-68.

[17] Stubbs N, Torpunuri VS, Lytton RL, Magnuson AH., A methodology to identify material properties in pavements modeled as layered viscoelastic half-spaces, In: Von Quintus HL, Bush AJ, Baladi GY, editors, NDT of pavements and backcalculation of moduli, 2. Special technical publication, STP 1198. Pennsylvania: ASTM Publication; 1994. p. 159-69.

[18] Jemioło S., Al-Garassi A.N.A., Generalized Boussinesq's problem for linear viscoelasticity materials, in edited by W. Szcześniak Theoretical Foundations of Civil Engineering, Polish-Ukrainian-Lithuanian Transactions, Warsaw-Vilnius, 97-104, ISBN 5-77638880-5, 2006.

[19] Szcześniak W., A Dynamic Problem of Elastic Beam on Maxwell's Foundation, Scientific Works of Warsaw University of Technology, z. 108, 1989.

[20] Lytton, R L; Uzan, J ; Fernando, E G; Roque, R ; Hiltunen, D ; Stoffels, S M, Devepopment and Validation of Performance Prediction Models and Specifications for Asphalt Binders and Paving Mixes, Strategic Highway Research Program, National Research Council, Washington, DC 1993. 